\title{
Self-redirection of tearing edges in graphene: Tight-binding molecular dynamics simulations
}

\author{
Takazumi Kawai, ${ }^{1, *}$ Susumu Okada, ${ }^{2,3}$ Yoshiyuki Miyamoto, ${ }^{1,3}$ and Hidefumi Hiura ${ }^{1}$ \\ ${ }^{1}$ Nano Electronics Research Laboratories, NEC Corporation, 34 Miyukigaoka, Tsukuba 305-8501, Japan \\ ${ }^{2}$ Institute of Physics and Center for Computational Science, University of Tsukuba, 1-1-1 Tennodai, Tsukuba 305-8571, Japan \\ ${ }^{3}$ CREST, Japan Science and Technology Agency, 4-1-8 Honcho, Kawaguchi, Saitama 332-0012, Japan
}

(Received 8 March 2009; published 1 July 2009)

\begin{abstract}
The narrower the width of the graphene, the higher the impact of the atomic edge structure on its electronic properties. Here, we have performed computer simulations for the mechanical tearing of graphene to form a graphene nanoribbon (GNR) and identified the atomic edge structures. The simulations clearly show that shear force applied to graphene results in armchair edge structures, independent of the tearing direction. Even if the tearing is initiated along the zigzag direction, the initial zigzag edge structures are immediately followed by the armchair edges. Thus, the GNRs with the atomically flat armchair edges can be formed through a wide range of conditions, which is essential for the production of low-dimensional nanodevices with the same specifications.
\end{abstract}

DOI: 10.1103/PhysRevB.80.033401

Graphene has regained much attention since its unique electronic properties were experimentally revealed, ${ }^{1-4}$ confirming its potential applications for nanoscale devices. ${ }^{5}$ Pseudo two-dimensional (2D) graphene differs significantly from bulk three-dimensional (3D) graphite especially in regard to the electronic properties. The presence of edges in graphene ribbons, which give rise to localized $\pi$ states, plays a key role in determining the characteristic graphene properties. ${ }^{6-10}$ The theoretical calculations suggest that the electronic properties are largely dependent on the atomic edge geometries for thinner graphenes which can be either armchair or zigzag. ${ }^{11}$ At the same time for electronic applications, atomically flat edges are preferred over rough ones to avoid uncontrollable scattering of conducting carriers.

Recently, narrow GNRs with widths below $10 \mathrm{~nm}$ were obtained experimentally by mechanical breaking of suspended graphene sheets under sonication. ${ }^{12}$ Massive fabrication of atomically flat edge GNRs was reported opening the door to electronic applications. ${ }^{12}$ Yet, the identification of the atomic edge structure is still difficult in the experiment. The static calculations of edge structures showed that the armchair type is more stable than the zigzag one. ${ }^{13,14}$ However, it is hard to imagine the appearance of armchair edge when tearing occurs in the zigzag direction. Indeed, the most stable structure does not always appear for dynamical formation processes in general. ${ }^{15}$ Once metastable atomic configurations have been realized, the appearance of more stable configurations must take time because of the presence of activation barriers in between the metastable and stable configurations. Beyond the static energy differences, the direct molecular dynamics (MD) simulations are important because the mechanical tearing off of graphene is basically an irreversible reaction and therefore it can provide insight into the dynamics of tearing and the resulting GNRs formed.

Our tight-binding MD simulations show that the mechanical tearing processes with a shearing force along armchair direction succeed in forming armchair edges without any defects. However tearing along the zigzag direction fails to form a zigzag edge and surprisingly results in the formation of armchair-edge structures, instead. The present findings are understood by the behavior of valence electrons which escape from the broken bond at the tearing front into the edge
PACS number(s): 61.48.De, 63.22. $-\mathrm{m}$, 73.22. $-\mathrm{f}$

states formed at the cutting gap. The anisotropy in the cutting direction can clearly explain the formation of atomically flat edge structures ${ }^{1,9,14}$ and GNRs with thinner width. ${ }^{12}$

We performed tight-binding MD simulations ${ }^{16}$ under constant temperature ${ }^{17,18}$ to simulate the tearing of graphene sheets and clarify the formation process of atomic edge structures. The tight-binding molecular dynamics method was already developed for the research such as molecular dynamics studies of the liquid phase of carbon and also for the structures of small carbon clusters. In fact, the method was successfully applied to GNRs (Refs. 13 and 19) as well as the defect formations and its diffusion on graphene $s p^{3}$ network, which include the various bond breaks and bond formations during those reactions. ${ }^{20,21}$ We therefore believe that the current tight-binding MD simulations can express behaviors of valence electrons, which is a key factor in determining edge structure appearing through MD simulations. We neglected the spin degrees of freedom in our simulations since the energy difference by considering the spin degrees of freedom is on the order of $10 \mathrm{meV}$ per edge atom due to the first-principles self-consistent pseudopotential method, ${ }^{11}$ which is within the energy fluctuation during the MD simulations.

We chose a graphene sheet with square shape, which consisted of 520 carbon atoms as shown in Figs. 1(a) and 1(b). We applied the surface-normal shearing force to demonstrate the graphene edge formations by tearing. ${ }^{22}$ One of the edges of the square was chosen for the shearing forces. To initiate the tearing of graphene sheets, we removed two or three atoms at the shear force boundary and broke some bonds. The constrained atoms at the graphene edge indicated by the green spheres (gray spheres near the top arrows) in Figs. 1(a) and 1(b) are moved in the opposite direction with a constant velocity perpendicular to the original graphene surface $(z$ direction) and they can move freely along surface parallel directions. Thus, the force acting on the tearing head does not exactly surface normal but has some flexibility since the graphene interface between edge constrained atoms and tearing head easily changes the direction of the force acting on the tearing head, especially when the tearing head is away from the constrained edge atoms after the tearing reaction proceeds. ${ }^{23}$ The velocity of constrained atoms was varied 


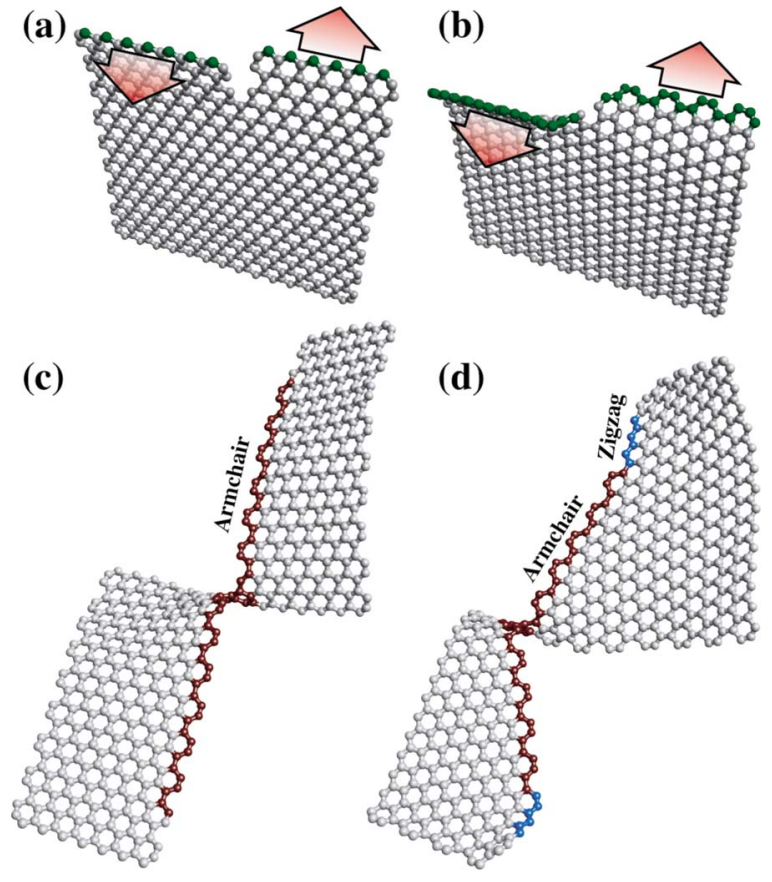

FIG. 1. (Color online) The initial atomic configurations for the graphene tearing along (a) the armchair, (b) the zigzag directions, and the resulting edge structures after tearing along (c) the armchair or (d) the zigzag directions. The green spheres (gray spheres near the top arrows) in (a) and (b) correspond to the constrained carbon atoms to tear the graphene sheet and others are carbon atoms without any constraints. The red/blue (dark gray/gray) spheres in (c) and (d) along the cut edges indicate carbon atoms forming lines of armchair/zigzag edges.

from 0.25 to $1.0 \mathrm{~nm} / \mathrm{ps}$. The simulations were done under two different constant temperatures, 300 and $1000 \mathrm{~K}$. Many MD simulations were done with different set of randomized velocities according to the temperatures.

We consider that our simulation results shown below will also be applicable for the formation of multilayered GNRs observed in the recent experiment ${ }^{12}$ since the interlayer interaction is too small to affect the formation of atomic edge structures. The multilayered GNRs can be formed when the initial cutting points are close to each other among layers and the direction of tearing is the same. Otherwise the layers are broken apart.

The simulations clearly showed the significance of armchair edge formation rather than zigzag one, independent of the initial direction of tearing. The snap shots of the simulations for the mechanical tearing along the armchair edge and the zigzag edge are shown in Figs. 1(c) and 1(d), respectively. Here, the velocity to separate the constrained atoms to opposite directions is $0.5 \mathrm{~nm} / \mathrm{ps}$ each at a temperature of 300 $\mathrm{K}$. When we set the tearing direction along the armchair as shown in Fig. 1(a), the resulting structure is armchair without any defects. There were two alternative bonds which were geometrically equivalent at the local atomic configuration and were extended almost equally until the saddle point configuration. Even if we tear off the graphene sheet along zigzag edge [see Fig. 1(b)], the armchair edge structures appeared after forming only a few zigzags for the condition above [see Fig. 1(d)]. Since the constraint effect of the tear-
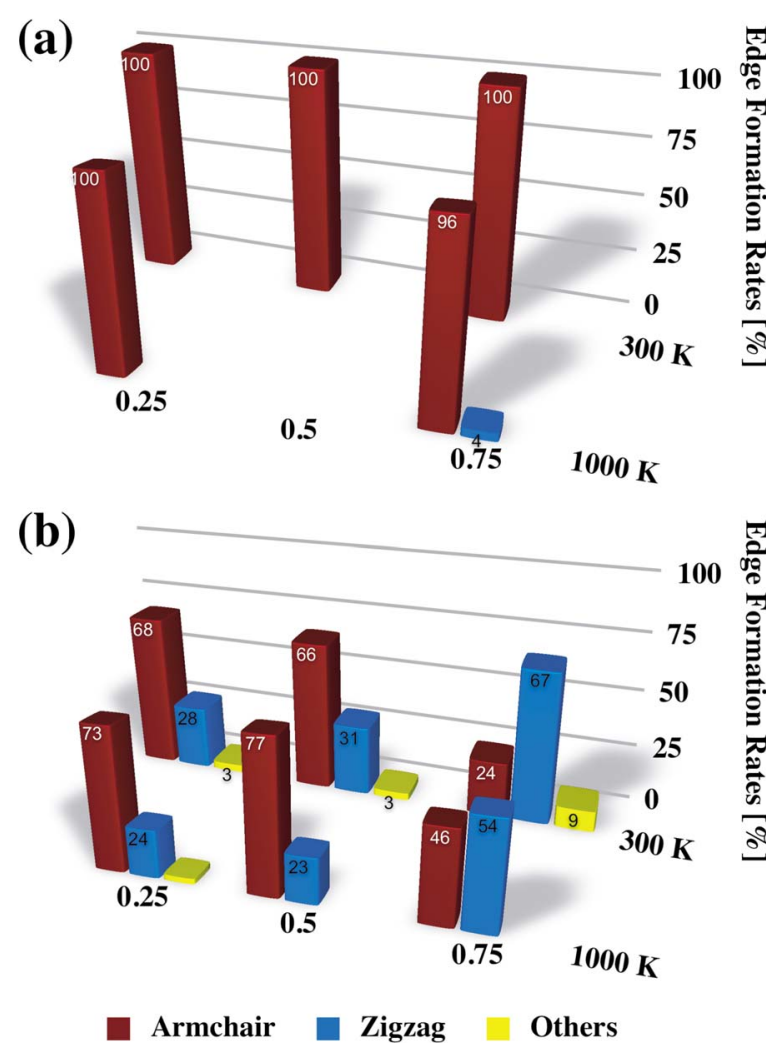

FIG. 2. (Color online) The bar graphs for the rates of specific edge length appeared after the tearing of a graphene sheet along (a) armchair edge and (b) zigzag edge depending on temperatures and speeds for the tearing.

ing direction should be reduced as the tearing proceeds, the formation of armchair edge will be kept for a larger graphene sheet.

In order to get statistical trend, we performed several MD simulations of graphene tearing with two different temperatures and three tearing speeds by changing the random velocities which were generated according to the initial temperatures. The ratios of length for armchair, zigzag, and other edge structures at the resulting cut edges are shown in Fig. 2 as a function of the temperature and the tearing speed. When the tearing direction is set along armchair direction, armchair edge structure appears dominantly as shown in Fig. 2(a). Even for the tearing along zigzag edge, the fraction of armchair edge structures is higher than the zigzag edge except for the fastest tearing speed. If the simulations are done by using larger graphene sheets, the ratios of armchair edge should be increased since the cutting line along armchair direction does not return back to zigzag direction.

Higher temperatures enhance the formation of armchair edge slightly, while higher tearing speed tends to extend the initial zigzag region. Then, a line of zigzag edge structure might appear under special tearing conditions at fairly fast tearing speeds at lower temperatures. We also found a peculiar edge structure with a tearing velocity of $0.5 \mathrm{~nm} / \mathrm{ps}$ with lower temperature condition. The structure is similar to the Klein edge structure ${ }^{24}$ and is immediately reconstructed to a 5 -membered ring termination. We found that the electronic ground state of the GNRs with this peculiar edge termination 
is ferromagnetic by first-principles electronic-structure calculations in the framework of the density functional theory using the local spin density approximation. ${ }^{25}$ We believe that the tearing speeds coincide with the local vibrational frequency and thereby lower the effective barriers to break particular C-C bonds which enhance the formation of these edge structures.

The mechanisms determining the edge structure are shown for the tearing along armchair direction [Figs. 3(a)-3(c)] and zigzag direction [Figs. 3(d)-3(f)]. The locally equivalent two bond types appear at the tearing front. Here, the choice of bond breaking indicated by " $A$ " or " $Z$ " in Fig. 3(a) develops the formation of the successive armchair edge or initiates the subsequent zigzag edge structures, respectively. The mechanical tension applied on the graphene is concentrated on the two bonds at the tearing front, and these bonds gradually stretch as the tension applied on the graphene increase. The two bonds are elongated equally up to $\sim 1.7 \AA$ and one of the bonds is disconnected beyond the saddle point configuration. Here, the electrons in the vicinity of tearing front lift their energy levels above the Fermi level $\left(\varepsilon_{f}\right)$. Therefore they must escape into one of the refuge electronic states associated with either armchair edge or zigzag edge through the thermal vibrations. When the electronic occupation of bond $A$ is reduced by the elongation of the bond under tension, the neighboring $\mathrm{C}-\mathrm{C}$ bond accepts the excess electrons and the process finally leads to the formation of triple-bond like configuration indicated by " $T$ " in Fig. 3(b). The triple-bond like configuration reminds us of the structure of a benzene. The corresponding energy levels of the triplebond like electronic states are about $2 \mathrm{eV}$ below $\varepsilon_{f} .{ }^{13}$ On the other hand, the excess electrons by the elongation of the bond $Z$ have no such stable refuge state. The refuge states for excess electrons below $\varepsilon_{f}$ significantly lower the reaction barrier to break the bond $A$ comparing to the barrier for the bond $Z$ breaking. After the breaking of the bond $A$ a similar configuration appears again at the tearing front. The succession of similar dynamics results in a formation of armchair edge structure as shown in Fig. 1(c).

In the case of tearing along the zigzag direction [Fig. $3(\mathrm{~d})$ ], there are one representative breaking bond $(Z)$ to make zigzag edge structure and two equivalent bonds ( $A$ and $\left.A^{\prime}\right)$ to make armchair edge. The tension applied on the graphene is also concentrated on these three bonds at the tearing front. In the initial few steps, the constrained edge atoms concentrate the tension for the tearing on the $Z$ bond forming zigzag edge structures. However, as the tearing front moves away from the graphene edge, the concentration of tension reaches the next bond $A$ in Fig. 3(d). Then the choice of the breaking bond is similar to the procedure for tearing along armchair direction. Again, the valence electrons at the tearing front can escape into the triple-bond like electronic structure whose energy level is below $\varepsilon_{f}$. Then the edge structure of tearing front switches from zigzag to armchair after the formation of the triple-bond like configuration $T$ shown in Fig. 3(e). The local atomic configuration in Fig. 3(f) for the next tearing steps becomes similar to those for the tearing along the armchair direction.

In the above simulations, we have concentrated on the simulations for the rectangle graphene sheet with the same
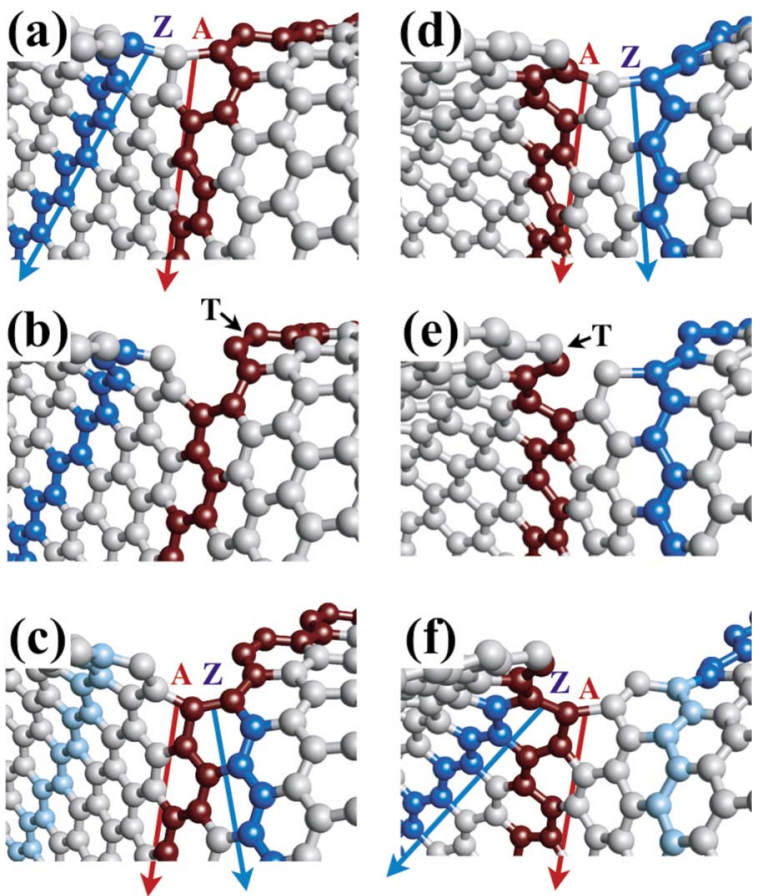

FIG. 3. (Color online) The snapshots for graphene tearing along (a)-(c) armchair direction and (d)-(f) zigzag direction. All the spheres represent carbon atoms and lines of red/blue (dark gray/ gray) spheres indicate the expected atomic edge structures for armchair/zigzag. The letters " $A$ " and " $Z$ " indicate the characteristic bonds at the tearing head to determine the edge structure and red (dark gray) and blue (gray) arrows indicate the directions to form armchair edge and zigzag edge, respectively. The triple-bond like structure appeared for the formation of armchair edge is indicated by " $T$."

size. The simulations show that the dynamics and resulting structures are independent of whether the tearing front is at the center or near the edge of the graphene patch during each of the tearing simulations. This fact indicates that the size of the system is large enough along the tearing direction.

To check the width-dependence of the graphene tearing, we also tested the formation of the ribbon structures by tearing along with two cutting lines on the graphene sheet, where the graphene sheet consists of 534 (540) carbon atoms for the formation of armchair (zigzag) ribbons, and some of the edge carbon atoms are removed to initiate the formation of the ribbons. The periodic boundary condition is applied along the width direction to mimic larger graphene sheet. The graphene ribbons with armchair edges were successfully formed by the tearing simulations. For the reaction along the zigzag direction, we could not obtain the ribbon structures, but just small patches.

The effects of the ambient gas molecules are one of the interesting topics, which might affect the resulting atomic structures of the edges. The tearing reaction is sequence of individual $\mathrm{C}-\mathrm{C}$ bond breaking, where the local electronic states near the sigma bond and nearest triple-bond like states play important roles. Thus, the edge terminations only near the reaction head possibly affect the resulting edge structures.

However, the probability of reaction between ambient gas 
molecules and reaction head is rather small in usual. For example, the number of oxygen molecules to pass through the region near the tearing head area $\left(1 \mathrm{~nm}^{3}\right)$ is order of $10^{4}$ molecules per second under the atmospheric condition from the simplest consideration. Thus, the average interval for a single molecule to visit the region is $\sim 10^{-4} \mathrm{~s}$. For tearing speed of $\sim 1 \mathrm{~cm} / \mathrm{s}$, the next bond breaking is already proceeded within $\sim 2.5 \times 10^{-8} \mathrm{~s}$. Furthermore, the reaction rate for the edge and atmospheric molecules would be sufficiently small. Then, even under presence of atomic or molecular species, the active tearing front should be free from chemical reactions during the tearing reaction under usual conditions.

We have demonstrated the dominance of armchair edge in GNR formation by performing tight-binding MD simulations. If we compare our results with experiments of micromechanical exfoliation of bulk graphite, the velocity to tear the graphene should be faster than experiments. Yet our simulations show a clear trend of dominant formation of the armchair edge in slower shearing velocities. We can therefore conclude that the thinner GNRs obtained in experiments should have armchair edge structures. Even if the shearing velocities for the sonochemistry and ultrahot gas bubbles involved in sonication are faster than micromechanical exfo- liation of graphite, we expect that the speed is not enough to form zigzag edge or other edge structures suggested in our simulations. This is the reason for the flat and parallel cutting edges of GNRs obtained in experiment. ${ }^{12}$ Thus, the edge structures of GNRs recently obtained in experiment must be armchair. In contrast, the formation of zigzag edge is very difficult. Here, the termination-free edge structures obtained from the graphene tearing might be reconstructed to different edge structures after the enough annealing time. ${ }^{26,27}$

For the formation of zigzag edge one can try faster tearing speed more than $1 \mathrm{~nm} / \mathrm{ps}$, which might be realized in sonication with the optimized solvents and/or polymer functionalizations. Chemical doping might be another possible way to change the resulting edge structures. The electronic states at the tearing front for the saddle-point configuration often appear at the Fermi level and the change in the occupation of these states by doping either donor or acceptor would alter the robustness of $\mathrm{C}-\mathrm{C}$ bond at the tearing front. ${ }^{28}$ Recently, Girit et al. ${ }^{29}$ discuss the stability of zigzag structure at the graphene hole edge.

We thank Thomas Ebbesen for fruitful discussions and comments. Part of the calculations were performed on the SX Supercomputer System at the NEC Fuchu Plant.
*Corresponding author. takazumi-kawai@mua.biglobe.ne.jp

${ }^{1}$ A. K. Geim and K. S. Novoselov, Nature Mater. 6, 183 (2007).

${ }^{2} \mathrm{Ph}$. Avouris, Z. Chen, and V. Perebeinos, Nat. Nanotechnol. 2, 605 (2007).

${ }^{3}$ K. S. Novoselov, A. K. Geim, S. V. Morozov, D. Jiang, Y. Zhang, S. V. Dubonos, I. V. Grigorieva, and A. A. Firsov, Science 306, 666 (2004).

${ }^{4}$ K. S. Novoselov, A. K. Geim, S. V. Morozov, D. Jiang, M. I. Katsnelson, I. V. Grigorieva, S. V. Dubonos, and A. A. Firsov, Nature (London) 438, 197 (2005).

${ }^{5}$ T. Ebbesen, H. Hiura, and K. Tanigaki, Japanese Patent 6-257019, 1994.

${ }^{6}$ M. Fujita, K. Wakabayashi, K. Nakada, and K. Kusakabe, J. Phys. Soc. Jpn. 65, 1920 (1996).

${ }^{7}$ K. Nakada, M. Fujita, G. Dresselhaus, and M. S. Dresselhaus, Phys. Rev. B 54, 17954 (1996).

${ }^{8}$ K. Wakabayashi, Phys. Rev. B 64, 125428 (2001).

${ }^{9}$ Y.-W. Son, M. L. Cohen, and S. G. Louie, Nature (London) 444, 347 (2006).

${ }^{10}$ Y. Niimi, T. Matsui, H. Kambara, K. Tagami, M. Tsukada, and H. Fukuyama, Phys. Rev. B 73, 085421 (2006).

${ }^{11}$ Y.-W. Son, M. L. Cohen, and S. G. Louie, Phys. Rev. Lett. 97, 216803 (2006).

${ }^{12}$ X. Li, X. Wang, L. Zhang, S. Lee, and H. Dai, Science 319, 1229 (2008).

${ }^{13}$ T. Kawai, Y. Miyamoto, O. Sugino, and Y. Koga, Phys. Rev. B 62, R16349 (2000).

${ }^{14}$ S. Okada, Phys. Rev. B 77, 041408(R) (2008).

${ }^{15}$ H. Hiura, T. W. Ebbesen, J. Fujita, K. Tanigaki, and T. Takada, Nature (London) 367, 148 (1994).

${ }^{16}$ C. H. Xu, C. Z. Wang, C. T. Chan, and K. M. Ho, J. Phys.: Condens. Matter 4, 6047 (1992).

${ }^{17}$ S. Nosé, J. Chem. Phys. 81, 511 (1984).
${ }^{18}$ W. G. Hoover, Phys. Rev. A 31, 1695 (1985).

${ }^{19}$ T. Kawai, S. Okada, Y. Miyamoto, and A. Oshiyama, Phys. Rev. B 72, 035428 (2005).

${ }^{20}$ C. Z. Wang and K. M. Ho, Phys. Rev. Lett. 71, 1184 (1993).

${ }^{21}$ G.-D. Lee, C. Z. Wang, J. Yu, E. Yoon, and K. M. Ho, Phys. Rev. Lett. 91, 265701 (2003).

${ }^{22}$ The surface-normal shearing force corresponds to the mechanical exfoliation of the graphene sheet from the graphite, where the scotch tape peels the layers of graphene from the graphite with the surface-normal force. We expect that the tearing process is important for the graphene formation via ultrasonication although the process is not clear.

${ }^{23}$ We also performed the simulations for graphene tearing with the surface parallel tension. Here, the gravity point of constrained atoms, which initially has the similar geometries as those in the previous simulations are separated in plane. The tearing head in the simulations never goes straight and the reaction just tears off small graphitic patches. Then, we could not get any straight edges, which is different from the corresponding experimental results. We, therefore, consider that the tearing with surfaceparallel tension never contributes to the formation of the ribbon structures or rather straight graphene-edge structures.

${ }^{24}$ D. J. Klein and L. Bytautas, J. Phys. Chem. A 103, 5196 (1999).

${ }^{25}$ S. Okada, K. Nakada, K. Kuwabara, K. Daigoku, and T. Kawai, Phys. Rev. B 74, 121412(R) (2006).

${ }^{26}$ P. Koskinen, S. Malola, and H. Hakkinen, Phys. Rev. Lett. 101, 115502 (2008).

${ }^{27}$ T. Wassmann, A. P. Seitsonen, A. M. Saitta, M. Lazzeri, and F. Mauri, Phys. Rev. Lett. 101, 096402 (2008).

${ }^{28}$ T. Kawai and Y. Miyamoto, Chem. Phys. Lett. 453, 256 (2008).

${ }^{29}$ C. O. Girit, J. C. Meyer, R. Erni, M. D. Rossell, C. Kisielowski, L. Yang, C.-H. Park, M. F. Crommie, M. L. Cohen, S. G. Louie, and A. Zettl, Science 323, 1705 (2009). 